UvA-DARE (Digital Academic Repository)

\title{
Physical mechanisms for the variable spin-down and light curve of SGR 1900+14
}

Thompson, C.; Duncan, R.C.; Woods, P.M.; Kouveliotou, C.; Finger, M.H.; van Paradijs, J.A.

Published in:

Astrophysical Journal

DOI:

$10.1086 / 317072$

Link to publication

Citation for published version (APA):

Thompson, C., Duncan, R. C., Woods, P. M., Kouveliotou, C., Finger, M. H., \& van Paradijs, J. A. (2000).

Physical mechanisms for the variable spin-down and light curve of SGR 1900+14. Astrophysical Journal, 543, 340. https://doi.org/10.1086/317072

\section{General rights}

It is not permitted to download or to forward/distribute the text or part of it without the consent of the author(s) and/or copyright holder(s), other than for strictly personal, individual use, unless the work is under an open content license (like Creative Commons).

\section{Disclaimer/Complaints regulations}

If you believe that digital publication of certain material infringes any of your rights or (privacy) interests, please let the Library know, stating your reasons. In case of a legitimate complaint, the Library will make the material inaccessible and/or remove it from the website. Please Ask the Library: https://uba.uva.nl/en/contact, or a letter to: Library of the University of Amsterdam, Secretariat, Singel 425, 1012 WP Amsterdam, The Netherlands. You will be contacted as soon as possible. 
The Astrophysical JournaL, 543:340-350, 2000 November 1

(C) 2000. The American Astronomical Society. All rights reserved. Printed in U.S.A.

\title{
PHYSICAL MECHANISMS FOR THE VARIABLE SPIN-DOWN AND LIGHT CURVE OF SGR 1900+14 \\ Christopher Thompson, ${ }^{1}$ Robert C. Duncan, ${ }^{2}$ Peter M. Woods, ${ }^{3,4}$ Chryssa Kouveliotou,, 4 MARK H. Finger, ${ }^{4,5}$ AND JAN VAN PARAdiJs ${ }^{3,6}$ \\ Received 1999 August 7; accepted 2000 June 12
}

\begin{abstract}
We consider the physical implications of the rapid spin-down of soft gamma repeater SGR $1900+14$ reported by Woods and colleagues in 1999. During an 80 day interval between 1998 June and the large outburst on 1998 August 27, the mean spin-down rate increased by a factor of 2.3, resulting in a positive period offset of $\triangle P / P=10^{-4}$. A radiation-hydrodynamical outflow associated with the August 27 event could impart the required torque, but only if the dipole magnetic field is stronger than $\sim 10^{14} \mathrm{G}$ and the outflow lasts longer and/or is more energetic than the observed X-ray flare. A positive period increment is also a natural consequence of a gradual, plastic deformation of the neutron star crust by an intense magnetic field, which forces the neutron superfluid to rotate more slowly than the crust. Sudden unpinning of the neutron vortex lines during the August 27 event would then induce a glitch opposite in sign to those observed in young pulsars, but of a much larger magnitude as a result of the slower rotation.

The change in the persistent X-ray light curve following the August 27 event is ascribed to continued particle heating in the active region of that outburst. The enhanced X-ray output can be powered by a steady current flowing through the magnetosphere, induced by the twisting motion of the crust. The long-term rate of spin-down appears to be accelerated with respect to a simple magnetic dipole torque. Accelerated spin-down of a seismically active magnetar will occur when its persistent output of Alfvén waves and particles exceeds its spin-down luminosity or if particle flows modulate the ratio of conduction to displacement currents in the outer magnetosphere. We suggest that SGRs experience some episodes of relative inactivity, with diminished $\dot{P}$, and that such inactive magnetars are observed as anomalous X-ray pulsars (AXPs). The reappearance of persistent X-ray emission from SGR 1900+14 within one day of the August 27 event provides strong evidence that the persistent emission is not powered by accretion.
\end{abstract}

Subject headings: pulsars: general — stars: individual (SGR 1900+14) - X-rays: bursts

\section{INTRODUCTION}

The soft gamma repeaters (SGRs) are sources of very intense X-ray flares, the large majority of which have a very short duration of $\sim 0.1 \mathrm{~s}$ (Gogus et al. 1999, 2000). They are best known for two giant flares: the first on 1979 March 5 from a source in the Large Magellanic Cloud, and the second on 1998 August 27 from SGR $1900+14$. These outbursts are almost carbon copies of each other. Each was initiated by a very intense pulse lasting $0.2-0.4 \mathrm{~s}$. The last part of each burst involved a train of large-amplitude pulsations, with 8.0 and $5.16 \mathrm{~s}$ periodicities, respectively (Mazets et al. 1979, 1999; Hurley et al. 1999a; Feroci et al. 1999). The enormous peak luminosity $\left(\sim 3-10 \times 10^{6}\right.$ times the Eddington luminosity of a neutron star for the March 5 event) and huge energy ( $E \sim 1 \times 10^{44}$ ergs for the August 27 event) of these outbursts point directly to the presence of very intense magnetic fields, $B>10 B_{\mathrm{QED}}=4.4 \times 10^{14} \mathrm{G}$ (Thompson \& Duncan 1995, hereafter TD95). Strong support for this magnetar model came from the detection of

\footnotetext{
${ }^{1}$ Department of Physics and Astronomy, University of North Carolina, Philips Hall, Chapel Hill, NC 27599-3255.

2 Department of Astronomy, University of Texas, RLM 15.308, Austin, TX 78712-1083.

${ }^{3}$ Department of Physics, University of Alabama in Huntsville, Huntsville, AL 35899.

${ }^{4}$ NASA Marshall Space Flight Center, SD50, Huntsville, AL 35812.

${ }^{5}$ Universities Space Research Association.

${ }^{6}$ Astronomical Institute "Anton Pannekoek," University of Amsterdam, 403 Kruislaan, 1098 SJ Amsterdam, NL.
}

a $7.47 \mathrm{~s}$ spin period and large period derivative corresponding to $P / \dot{P}=3 \times 10^{3} \mathrm{yr}$ in SGR $1806-20$ (Kouveliotou et al. 1998), followed shortly by the detection of a $5.16 \mathrm{~s}$ spin period (Hurley et al. 1999b) and rapid spin-down $(P / \dot{P}=3$ $\times 10^{3} \mathrm{yr}$; Kouveliotou et al. 1999) in SGR $1900+14$. Indeed, the large-amplitude pulsations subsequently detected in the August 27 event had the same period as was observed in the persistent emission of SGR $1900+14$.

Woods et al. (1999b, hereafter Paper I) have shown that over the period 1996 September-1999 May, the spin-down history of SGR $1900+14$ is generally smooth, with an average rate of $6 \times 10^{-11} \mathrm{~s} \mathrm{~s}^{-1}$. However, during an 80 day interval starting in 1998 June, which contains the extremely energetic August 27 flare, the average spin-down rate of SGR $1900+14$ increased by a factor of $\sim 2.3$. The sampling of the period history of SGR $1900+14$ is insufficient to distinguish between a long-term (i.e., 80 days) increase of the spin-down rate to an enhanced value and a sudden increase (a "braking" glitch) in the spin period connected with the luminous August 27 flare.

In this paper we investigate several physical processes that may generate a positive period increment of the observed magnitude $\left(\Delta P / P \sim 10^{-4}\right)$ directly associated with the August 27 flare. We focus on two mechanisms: (1) a wind of particles and MHD waves coinciding with the period of hyper-Eddington radiative flux, and (2) an exchange of angular momentum between the crustal neutron superfluid and the rest of the neutron star. We show that both models point to the presence of an intense magnetic field. The change in the persistent pulse profile of 
SGR $1900+14$ following the August 27 outburst is considered and related to continuing particle output in the active region of the burst. We also consider mechanisms that could drive the (nearly) steady spin-down observed in both SGRs and anomalous X-ray pulsars (AXPs), as well as departures from uniform spin-down.

\section{BRAKING DRIVEN BY A PARTICLE OUTFLOW}

The two giant outbursts appear to have been powered by the sudden deposition of a huge amount of energy, 1$4 \times 10^{44}$ ergs (TD95). Part of this energy escaped directly as an expanding $e^{ \pm}$fireball that was detected as the very intense initial pulse of $\gamma$-rays. In the August 27 event, this pulse had a duration of $\sim 0.35 \mathrm{~s}$ and energy greater than $7 \times 10^{43}(D / 10 \mathrm{kpc})^{2}$ ergs (Mazets et al. 1999). The remainder of the energy appears to have been trapped close to the neutron star in the form of a thermal fireball (TD95; Hurley et al. 1999a; Feroci et al. 1999) and radiated gradually during the extended phase of large-amplitude oscillations. The radiative flux decreased from $1 \times 10^{42}(D / 10 \mathrm{kpc})^{2} \mathrm{ergs}$ $\mathrm{s}^{-1}$ over a period of $300 \mathrm{~s}$, during the latter part of the August 27 outburst (Mazets et al. 1999). The net energy in the tail, radiated in photons of energy greater than $15 \mathrm{keV}$, was $\sim 5 \times 10^{43}(D / 10 \mathrm{kpc})^{2}$ ergs.

Just as in the case of the March 5 burster, several arguments indicate the presence of a magnetic field stronger than $10^{14} \mathrm{G}$ in SGR $1900+14$ (TD95). Not only can such a field spin down the star to its observed $5.16 \mathrm{~s}$ period (Hurley et al. 1999b; Kouveliotou et al. 1999), but it can also power the burst by inducing a large-scale fracture of the neutron star crust. Indeed, only a fraction of $\sim 10^{-2}\left(B_{*} / 10 B_{\mathrm{QED}}\right)^{-2}$ of the external dipole magnetic energy must be tapped, where $B_{\mathrm{QED}} \equiv 4.4 \times 10^{13} \mathrm{G}$. This allows for individual SGR sources to emit $\gtrsim 10^{2}$ such giant flares over their $\sim 10^{4} \mathrm{yr}$ active lifetimes. More generally, any energy source that excites internal seismic modes of the neutron star must be combined with a magnetic field of this strength, if seismic energy is to be transported across the stellar surface at the (minimum) rate observed in the initial spike (see Thompson 2000; Feroci et al. 1999). A field stronger than $1.5 \times 10^{14}(E /$ $\left.6 \times 10^{43} \mathrm{ergs}\right)^{1 / 2}(\Delta R / 10 \mathrm{~km})^{-3 / 2}\left[\left(1+\Delta R / R_{*}\right) / 2\right]^{3} \mathrm{G}$ is also required to confine the energy radiated in the oscillatory tail (Hurley et al. 1999a), which maintained a very constant temperature even while the radiative flux declined by an order of magnitude (Mazets et al. 1999).

The radiative flux was high enough throughout the August 27 event that a large amount of baryonic plasma could have been advected out at relativistic speed. Indeed, the large amplitude of the X-ray pulsations detected during the latter part of the August 27 event requires strong collimation of the X-ray flux into narrow fans or beams that are swept past the line of sight as the source rotates. Electron scattering becomes strongly anisotropic in an intense background magnetic field, and there is a large inequality between the scattering cross sections of the two (linear) photon polarization modes (Herold 1979; Paczyński 1992). The cross section of the extraordinary mode ( $E$-mode, with $\left.\delta \boldsymbol{E} \cdot \boldsymbol{B}_{0}=0\right)$ is pushed far below Thomson, $\sigma(E) / \sigma_{\mathrm{T}}=$ $\left(\omega m_{e} c / e B\right)^{2}$, whereas the orthogonal ordinary mode $(O-$ mode) maintains $\sigma(O) \sim \sigma_{\mathrm{T}}$ except when it propagates almost parallel to $\boldsymbol{B}_{0}$. This allows the radiative flux of $E$-mode photons across a confining magnetic field to exceed greatly the classical Eddington flux from a neutron star
(TD95). However, photon splitting ${ }^{7}$ will convert a significant fraction of the $E$-mode radiative flux to the $O$-mode above an effective temperature of $\sim 11 \mathrm{keV}$ and ensure that the radiation and matter are hydrodynamically coupled near the stellar surface (TD95). Only a small fraction $\dot{M} c^{2} / L_{O}>\left(L_{O} / L_{\mathrm{Edd}}\right)^{-1}$ of the classical Eddington luminosity need be carried by matter rest energy to provide a large scattering depth to $O$-mode photons near the base of the outflow. The coupling between radiation and matter is also greatly enhanced by the rapid growth in the $E$-mode scattering opacity with distance from the surface of the neutron star, $\sigma(E) \propto B^{-2} \propto R^{6}$. As a result, a collimated flux of $E$-mode photons can escape only by forcing to the side matter suspended in the magnetosphere.

In the radiative model for SGR outbursts detailed in TD95, the surface of the neutron star is exposed to a trapped $e^{ \pm}-\gamma$-ray fireball. The temperature of this fireball greatly exceeds that of the neutron star crust and is $\sim 1$ $\mathrm{MeV}$ in the case of a giant outburst like the August 27 event. As the cooling fireball contracts in volume (at a rate greatly accelerated by the low $E$-mode opacity close to the star), the heated surface is exposed and drives a superEddington flux of photons. In such a situation, scattering of the $E$-mode to the $O$-mode will drive matter off the surface of the neutron star (Miller 1995; TD95). The strong magnetic tension allows a considerable mass of ejected material to accumulate further out in the magnetosphere during the burst, but the associated scattering depth cannot exceed $\tau_{\mathrm{T}} \sim 1$ outside a radius where the energy density of the freely streaming photons exceeds the dipole magnetic energy density,

$$
\frac{L_{\mathrm{X}}}{4 \pi R_{\mathrm{A}}^{2} c} \sim \frac{B_{*}^{2}}{4 \pi}\left(\frac{R_{\mathrm{A}}}{R_{*}}\right)^{-6}
$$

or equivalently

$$
\begin{aligned}
\frac{R_{\mathrm{A}}}{R_{*}} \sim\left(\frac{B_{*}^{2} R_{*}^{2} c}{L_{\mathrm{X}}}\right)^{1 / 4}= & 280\left(\frac{B_{*}}{10 B_{\mathrm{QED}}}\right)^{1 / 2} \\
& \times\left(\frac{\Delta E_{\mathrm{X}}}{10^{44} \mathrm{ergs}}\right)^{-1 / 4}\left(\frac{\Delta t_{\text {burst }}}{100 \mathrm{~s}}\right)^{1 / 4} .
\end{aligned}
$$

The radiation pressure acting on the suspended matter will overcome the dipole magnetic pressure at a radius $\geq R_{\mathrm{A}}$; the same is true for the ram pressure of matter streaming relativistically outward along the dipole field lines. Near this Alfvén radius, the outflow can spread over a large solid angle.

Let us now estimate the maximum angular momentum that could be carried off during the August 27 outburst. Photons scattering last at radius $R_{\mathrm{A}}$ and polar angle $\theta$ (or relativistic matter escaping the dipole magnetic field from the same position) will carry a specific angular momentum $\sim \Omega R_{\mathrm{A}}^{2} \sin ^{2} \theta$. The net loss of angular momentum corresponding to an energy release $\Delta E$ is

$$
I_{*} \Delta \Omega \simeq-\frac{\Delta E}{c^{2}} \Omega R_{\AA}^{2} \sin ^{2} \theta .
$$

An upper bound to the period increase, accumulated on a timescale $\Delta t_{\text {burst }}$, is obtained by assuming that the torque is

\footnotetext{
${ }^{7}$ The $O$-mode cannot split under the conditions appropriate to the photosphere of an SGR outburst (e.g., Thompson 2000).
} 
concentrated in the equatorial plane of the star:

$$
\begin{aligned}
\frac{\Delta P}{P} \simeq & \left(\Delta E \Delta t_{\text {burst }}\right)^{1 / 2} \frac{B_{*} R_{*}^{3}}{I_{*} c^{3 / 2}} \\
= & 8 \times 10^{-6}\left(\frac{\Delta E}{10^{44} \mathrm{ergs}}\right)^{1 / 2} \\
& \times\left(\frac{\Delta t_{\text {burst }}}{100 \mathrm{~s}}\right)\left(\frac{B_{*}}{10 B_{\mathrm{QED}}}\right) .
\end{aligned}
$$

The torque is negligible if the dipole field is in the range $B_{*} \sim 0.1 B_{\mathrm{QED}}$ typical of ordinary radio pulsars. Even for $B_{*} \sim 10 B_{\mathrm{QED}}$ this mechanism can induce $\Delta P / P \sim 1 \times 10^{-4}$ only if the outflow lasts longer than the observed duration of the oscillatory tail and/or carries more energy than the observed X-ray flare.

The release of $\sim 10^{44}$ ergs (comparable to the observed X-ray fluence) over $\sim 10^{4} \mathrm{~s}$ (a few hundred times the observed burst duration) would generate the observed torque, but extending the duration of the outflow to $\sim 10^{5} \mathrm{~s}$ would imply $\dot{P} \sim 1.3 \times 10^{-8}$ one day after the August 27 event, in contradiction with the measured value 200 times smaller. Note also that the short initial spike is expected to impart a negligible torque to the star. This is the basic reason that persistent fluxes of Alfvén waves and particles are more effective at spinning down a magnetar than are sudden, short bursts of equal fluence (Thompson \& Blaes 1998, hereafter TB98).

Hiding most of the August 27 burst output in Alfvén radiation would require that most of the energy was released in a low-frequency wave deep in the interior of the neutron star and then coupled on a long timescale $(>100 \mathrm{~s})$ to a magnetized outflow. That is because a high-frequency wave $\left(v \sim c / R_{*}\right)$ excited close to the star by reconnection would damp quickly through a turbulent cascade (TB98), with a high radiative efficiency. However, it is difficult to combine the rapid injection of energy in the initial spike with a long damping time for such an internal wave.

One might consider increasing the torque by increasing the inertia of the outflow, so that it moves subrelativistically at the Alfvén surface, at speed $V$. For a fixed kinetic luminosity, $\dot{E}=(1 / 2) \dot{M} V^{2}$, the Alfvén radius scales in proportion to $(V / c)^{1 / 4}$, and one finds

$$
\begin{aligned}
\frac{\Delta P}{P} \simeq 1 & \times 10^{-4}\left(\frac{\Delta E}{10^{44} \mathrm{ergs}}\right)^{1 / 2}\left(\frac{\Delta t_{\text {burst }}}{100 \mathrm{~s}}\right)^{1 / 2} \\
& \times\left(\frac{B_{*}}{10 B_{\mathrm{QED}}}\right)\left(\frac{V}{0.2 c}\right)^{-3 / 2} .
\end{aligned}
$$

However, the energy needed to lift this material from the surface of the neutron star exceeds $\Delta E=\int \dot{E} d t$ by a factor of $\sim 10(V / 0.2 c)^{-2}$ [assuming $G M_{*} /\left(R_{*} c^{2}\right)=0.2$ ]. This scenario therefore requires some fine-tuning, if the flow is to remain subrelativistic far from the neutron star.

Moreover, such a slow outflow is very thick to Thomson scattering and free-free absorption. The Thomson depth along a radial line through the outflow is

$$
\begin{aligned}
\tau_{\mathrm{T}}\left(R_{\mathrm{A}}\right)= & 10\left(\frac{\Delta E}{10^{44} \mathrm{ergs}}\right)^{5 / 4}\left(\frac{B_{*}}{10 B_{\mathrm{QED}}}\right)^{-1 / 2} \\
& \times\left(\frac{\Delta t_{\text {burst }}}{100 \mathrm{~s}}\right)\left(\frac{V}{c}\right)^{-13 / 4}
\end{aligned}
$$

at the Alfvén radius. The free-free optical depth is

$$
\tau_{\mathrm{ff}} \simeq \frac{\alpha_{\mathrm{em}} \bar{g}_{\mathrm{ff}}}{3^{1 / 2}(2 \pi)^{3 / 2}}\left(\frac{k T}{m_{e} c^{2}}\right)^{-1 / 2} \frac{\tau_{\mathrm{T}}^{2}(h c)^{3}}{\sigma_{\mathrm{T}} R(k T)^{3}} f\left(\frac{h v}{k T}\right),
$$

where

$$
f\left(\frac{h v}{k T}\right) \equiv\left(\frac{h v}{k T}\right)^{-3}\left(1-e^{-h v / k T}\right)
$$

and $\alpha_{\mathrm{em}}=1 / 137$ is the fine-structure constant. This becomes

$$
\begin{aligned}
\tau_{\mathrm{ff}}(R)= & 3 \times 10^{-2}\left(\frac{R}{R_{\mathrm{A}}}\right)^{-5}\left(\frac{\Delta P / P}{10^{-4}}\right)^{5 / 4}\left(\frac{\Delta E}{10^{44} \mathrm{ergs}}\right)^{1 / 2} \\
& \times\left(\frac{B_{*}}{10 B_{\mathrm{QED}}}\right)^{-16 / 3}\left(\frac{\Delta t_{\text {burst }}}{100 \mathrm{~s}}\right) f\left(\frac{h v}{k T}\right) .
\end{aligned}
$$

Here we have substituted the value of $V / c$ needed to generate the observed $\Delta P / P$. Notice that the magnetic dipole field and burst duration enter into $\tau_{\mathrm{ff}}$ with strong negative powers. The optical depth through a flow along rigid dipole magnetic field lines is $\tau_{\mathrm{T}}(R)=\left(R / R_{\mathrm{A}}\right)^{-2} \tau_{\mathrm{T}}\left(R_{\mathrm{A}}\right)$ at constant $V$.

This calculation indicates that the flow will be degraded to a blackbody temperature corresponding to an emission radius of $\sim 100 R_{*}=1000 \mathrm{~km}$, which is $\sim 1 \mathrm{keV}$ at a luminosity $\sim 10^{4} L_{\text {Edd }}$, far below the observed value (Mazets et al. 1999; Feroci et al. 1999). Note, however, that Inan et al. (1999) found evidence for an intense ionizing flux of soft $\mathrm{X}$-rays in Earth's ionosphere, coincident with the first second of the August 27 event. They fit these ionization data with an incident spectrum containing two thermal components, of temperatures 200 and $5 \mathrm{keV}$, and with the soft component carrying $80 \%$ of the energy flux at $5 \mathrm{keV}$. This model contrasts with the initial spectrum of the August 27 event measured by BeppoSAX, which contained a very hard power-law component $\left(v F_{v} \propto v^{1 / 2}\right.$; Feroci et al. 1999). The effects of pair creation on the ionization rate have yet to be quantified.

The four-pronged profile seen within the later pulses of the August 27 event (Feroci et al. 1999; Mazets et al. 1999) has a plausible interpretation in the magnetar model. The radiation-hydrodynamical outflow originates near the surface of the neutron star, where the opacity of X-ray photons moving across the magnetic field lines is smallest (TD95). This is the case even if the trapped $e^{ \pm}$fireball that powers the burst extends well beyond the stellar surface. In this model, the pattern of the emergent X-ray flux is a convolution of the multipolar structure of the stellar magnetic field, with the orientation of the trapped fireball. The presence of four X-ray "jets" requires that the trapped fireball connect up with four bundles of magnetic field lines extending to at least a few stellar radii.

\section{BRAKING VIA THE INTERNAL EXCHANGE OF ANGULAR MOMENTUM}

Now let us consider the exchange of angular momentum between the crustal superfluid neutrons and the rest of the magnetar. Because an SGR or AXP source is slowly rotating, $\Omega_{\mathrm{cr}} \sim 1$, the maximum angular velocity difference $\omega=$ $\Omega_{\mathrm{sf}}-\Omega_{\mathrm{cr}}$ that can be maintained between superfluid and crust is a much larger fraction of $\Omega_{\text {cr }}$ than it is in an ordinary radio pulsar and may even exceed it. At the same time, 
these sources are observed to spin down very rapidly, on a timescale comparable to young radio pulsars such as Crab or Vela. If the rotation of the superfluid were to lag behind the crust in the usual manner hypothesized for glitching radio pulsars, the maximum glitch amplitude would increase in proportion to the spin period (Thompson \& Duncan 1996, hereafter TD96; Heyl \& Hernquist 1999). One deduces $\Delta P / P \simeq-1 \times 10^{-5}$ by scaling to the largest glitches of the Crab pulsar and $\Delta P / P \simeq-1 \times 10^{-4}$ by scaling to Vela.

How would a glitch be triggered in a magnetar? A sudden fracture of the crust, driven by a magnetic field stronger than $\sim 10^{14} \mathrm{G}$, induces a horizontal motion at the Alfvén speed $V_{\mathrm{A}}=1.3 \times 10^{7}\left(B / 10 B_{\mathrm{QED}}\right)\left(\rho / 10^{14} \mathrm{~g} \mathrm{~cm}^{-3}\right)^{-1 / 2}$ $\mathrm{cm} \mathrm{s}^{-1}$ or higher. This exceeds the maximum velocity difference $V_{\text {sf }}-V_{\text {cr }}$ that can be sustained between superfluid and crust, before the neutron vortex lines unpin (e.g., Link, Epstein, \& Baym 1993). The internal heat released in a large flare such as the August 27 event is probably comparable to the external X-ray output, if the flare involves a propagating fracture of the neutron star crust. This heat is $\sim 100$ times the minimum energy of $\sim 10^{42}$ ergs that will induce a sudden increase in the rate of thermal vortex creep (Link \& Epstein 1996). For both reasons, giant flares from magnetars probably trigger the widespread unpinning of superfluid vortices in the crust and hence large rotational glitches. Magnetically driven fractures have also been suggested as the trigger for vortex unpinning in ordinary radio pulsars (Thompson \& Duncan 1993, hereafter TD93; Ruderman, Zhu, \& Chen 1998).

The observation of a period increase associated with the August 27 outburst leads us to reexamine whether the superfluid should, in fact, maintain a faster spin than the crust and charged interior of the star. Transport of superfluid vortices by thermal creep will cause the angular velocity lag $\omega$ to relax to its equilibrium value $\omega_{\infty}$ on a timescale

$$
t_{r}^{-1}=\left|\frac{\partial \Omega_{\mathrm{cr}}}{\partial t}\right|\left(\frac{\partial \ln V_{\mathrm{cr}}}{\partial \omega}\right)_{\omega_{\infty}},
$$

if the creep is driven primarily by spin-down (Alpar et al. 1984; Link et al. 1993). The partial derivative of the creep velocity $\partial V_{\mathrm{cr}} / \partial \omega$ depends mainly on temperature and density. As a result, this relaxation time is expected to be proportional to $t / \Omega_{\mathrm{cr}}$ at constant temperature. Comparing with a prompt (intermediate) relaxation time of $\sim 1$ day ( $\sim 1$ week) for glitches of the Crab pulsar $\left(t \simeq 10^{3} \mathrm{yr}\right.$; Alpar et al. 1996), one infers $t_{r} \sim 1$ (10) yr for a magnetar of spin period $6 \mathrm{~s}$ and characteristic age $P / \dot{P}=3000 \mathrm{yr}$.

The response of the crust to the evolving magnetic field is expected to be a combination of sudden fractures and plastic deformation. When the temperature of the crust exceeds $\sim 0.1$ of the melt temperature, it will deform plastically (Ruderman 1991). One deduces $T \simeq 2.4$ $\times 10^{8}\left(B_{\text {in }} / 10^{2} B_{\mathrm{QED}}\right)^{2} \mathrm{~K}$ for magnetars of age $\sim 10^{4} \mathrm{yr}$, where $B_{\text {in }}$ is the interior (rms) magnetic flux density (TD96; Heyl \& Kulkarni 1998). Plastic deformation is also expected when $B_{\text {in }}^{2} / 4 \pi>\mu$ in the deep crust (TD96). In a circumstance in which the magnetic field is transported through the stellar interior on a timescale shorter than the age of the star, departures from corotation between superfluid and crust are primarily due to advection of the superfluid vortices across the stellar surface by the deforming crust, not due to spin-down. (Recall the principal definition of a mag- netar: a neutron star in which magnetism, not rotation, is the dominant source of free energy.) If these deformations occur on a timescale much less than the spin-down age, they will control the equilibrium lag between the rotation of the superfluid and crust.

Indeed, the SGR bursts provide clear evidence for deformations on short timescales. More precisely, a large burst such as the August 27 event may be preceded (or followed) by an extended period of slow, plastic deformation. If the superfluid starts near corotation with the crust, this process will take angular momentum out of the superfluid and force its rotation to lag behind the rest of the star. A glitch triggered by a violent disturbance such as the August 27 event will then cause the neutron star crust to spin down.

The angular momentum of the thin shell of crustal superfluid can be expressed simply as

$$
J_{\mathrm{sf}}=\frac{\kappa}{2} M_{\mathrm{sf}} R_{*}^{2} \int_{-1}^{1} d(\cos \theta)\left(\cos ^{2} \theta\right) n_{V}(\theta),
$$

when the cylindrical density $n_{V}(\theta)$ of neutron vortex lines depends only on angle $\theta$ from the axis of rotation. Here $\kappa=h / 2 m_{n}$ is the quantum of circulation, and we neglect the rotational and magnetic deformations of the star. One observes from this expression that the outward motion of vortex lines reduces $J_{\text {sf }}$ because the weighting factor $\cos ^{2} \theta$ decreases with distance from the axis of rotation.

The simplest deformation of the neutron star crust, which preserves its mass and volume, involves a rotational twist of a circular patch through an angle $\Delta \phi$. Indeed, the stable stratification of the star (Reisenegger \& Goldreich 1992) forces the crust to move horizontally, parallel to the local equipotential surfaces. For this reason, one can neglect horizontal displacements of the crustal material that are compressible in the two nonradial dimensions. The patch has radius $a \ll R_{*}$ and is centered at an angle $\theta$ from the axis of rotation. The superfluid is assumed initially to corotate with the crust, $\Omega_{\mathrm{sf}}=\Omega_{\mathrm{cr}}$, everywhere within the patch, so that $n_{V}(\theta)=2 \Omega_{\mathrm{cr}} / \kappa$. As the patch is rotated, the number of vortex lines per unit surface area of crust is conserved. A piece of crust that moves from $\theta_{i}$ to $\theta_{f}$ ends up with a vortex density $n_{V}=\left(2 \Omega_{\text {cr }} / \kappa\right) \cos \theta_{i} / \cos \theta_{f}$. The vortex lines are squeezed together in a piece of the crust that moves away from the rotation axis and are spread apart if the movement is in the opposite direction. If the vortex density is smoothed out in azimuth following this process, the net decrease in the angular momentum of the superfluid is

$$
\frac{\Delta J_{\mathrm{sf}}}{J_{\mathrm{sf}}}=-\frac{3}{4}\left(\frac{a}{R_{*}}\right)^{4}(1-\cos \phi) \sin ^{2} \theta .
$$

Here $J_{\mathrm{sf}}=\frac{2}{3} M_{\mathrm{sf}} \Omega_{\mathrm{cr}} R_{*}^{2} \simeq 10^{-2} I_{*} \Omega_{\mathrm{cr}}$ is the total angular momentum of the crustal superfluid.

A transient, plastic deformation of the crust would induce a measurable spin-up of the crust by forcing the neutron superfluid farther from corotation with the crust. Such a gradual glitch would have the same negative sign as in ordinary radio pulsars but would not necessarily involve any sudden unpinning of the vortex lines. For example, rotation of a patch of radius $a=R_{*} / 3$ through an angle $\Delta \phi \sim 1$ radian would cause a period decrease $\Delta P / P=\Delta J_{\text {sf }} /$ $\left(I_{*}-I_{\mathrm{sf}}\right) \Omega_{*}=-4 \times 10^{-5}$. A transient spin-up of this magnitude may have been observed in the AXP source 1E $2259+586$ (Baykal \& Swank 1996). That excursion from a constant, long-term spin-down trend can be modeled with a 
glitch of amplitude $\Delta P / P \simeq-3 \times 10^{-5}$, although the $\mathrm{X}$-ray period observations are generally too sparse to provide a unique fit.

\section{THE LONG-TERM SPIN-DOWN OF SGRs AND AXPs}

Let us now consider the persistent spin-down rate of SGR $1900+14$ and its broader implications for the ages and spin-down histories of the SGR and AXP sources. Recall that the spin-down rate was almost constant at $\dot{P} \simeq$ $6.1 \times 10^{-11} \mathrm{~s} \mathrm{~s}^{-1}$ before 1998 May and after 1998 August 28 (Paper I). A 1997 May measurement of $P$ revealed a 5\% deviation from this trend, and larger variations in the "instantaneous" spin-down rate $(\sim 40 \%)$ were found by RXTE in 1996 September and 1998 May/June.

Another important constraint comes from the observed angular position of SGR $1900+14$. It lies just outside the edge of the $\sim 10^{4}$ yr old supernova remnant G42.8+0.6 (Hurley et al. 1994; Vasisht et al. 1994). A strong parallel can be drawn with SGR 0526-66, which also emitted a giant flare (on 1979 March 5) and is projected to lie inside, but near the edge of, supernova remnant (SNR) N49 in the Large Magellanic Cloud (Cline et al. 1982). The other known SGRs also have positions coincident with supernova remnants of comparable ages (Kulkarni \& Frail 1993; Kulkarni et al. 1994; Murakami et al. 1994; Hurley et al. 1999d, 1999e; Woods et al. 1999a; Smith, Bradt, \& Levine 1999). It seems very likely that these physical associations are real, so we will hereafter adopt the hypothesis that SGR $1900+14$ formed at the center of SNR G42.8+0.6. The implied transverse velocity is

$$
V_{\perp} \approx 3400\left(\frac{D}{7 \mathrm{kpc}}\right)\left(\frac{t}{10^{4} \mathrm{yr}}\right)^{-1} \mathrm{~km} \mathrm{~s}^{-1}
$$

(Hurley et al. 1996; Vasisht et al. 1994; Kouveliotou et al. 1999). Several mechanisms may impart large recoil velocities to newborn magnetars (Duncan \& Thompson 1992, hereafter DT92), but this very high speed indicates that an age much less than $1 \times 10^{4} \mathrm{yr}$ is unlikely.

In this context, the short characteristic spin-down age $t_{\mathrm{MDR}} \equiv P / 2 \dot{P} \sim 1340$ yr of SGR $1900+14$ gives evidence that the star is currently in a transient phase of accelerated spin-down (Kouveliotou et al. 1999). The almost identical spin-down age measured for SGR 1806-20 suggests that a similar effect is being observed in that source (Kouveliotou et al. 1998; see also Table 1). If each SGR undergoes accelerated spin-down during a minor fraction $\epsilon_{\text {active }} \sim P /$ $\dot{P} t_{\mathrm{SNR}} \sim 0.25$ of its life, then its true age increases to

$$
t=\epsilon_{\mathrm{active}}^{-1}\left(\frac{P}{\dot{P}}\right) \text {. }
$$

\subsection{Wind-aided Spin-down}

Seismic activity will accelerate the spin-down of an isolated neutron star, if the star is slowly rotating and strongly magnetized (TB98). Fracturing in the crust generates seismic waves that couple directly to magnetospheric Alfvén modes and to the relativistic particles that support the associated currents. The fractures are frequent and low energy $\left(\sim 10^{35}\right.$ ergs) when the magnetic field is forced across the crust by compressive transport in the core (TD96). When the persistent luminosity $L_{\mathrm{A}}$ of waves and particles exceeds the magnetic dipole luminosity $L_{\mathrm{MDR}}$ (as calculated from the stellar dipole field and angular velocity), the spindown torque increases by a factor of $\sim\left(L_{\mathrm{A}} / L_{\mathrm{MDR}}\right)^{1 / 2}$.

This result follows directly from our treatment of hydrodynamic torques in $\S 2$. Magnetic stresses force the relativistic wind to corotate with the star out to the Alfvén radius $R_{\mathrm{A}}$, which is determined by substituting $L_{\mathrm{A}}$ for $L_{\mathrm{X}}$ in equation (2):

$$
\frac{R_{\mathrm{A}}}{R_{*}}=1.6 \times 10^{4} L_{\mathrm{A} 35}^{-1 / 4}\left(\frac{B_{*}}{10 B_{\mathrm{QED}}}\right)^{1 / 2} .
$$

The torque then has the form $I \dot{\Omega}=-\Lambda \Omega\left(L / c^{2}\right) R_{\mathrm{A}}^{2}$, or equivalently

$$
\dot{P}=\Lambda \frac{B_{*} R_{*}^{3}}{I_{*}}\left(\frac{L_{\mathrm{A}}}{c^{3}}\right)^{1 / 2} P
$$

Here $\Lambda$ is a numerical factor of order unity that depends on the angle between the angular velocity $\Omega$ and the dipole magnetic moment $\boldsymbol{m}_{*}$. One finds $\Lambda \approx 2 / 3$ by integrating equation (3) over polar angle, under the assumption that $\Omega$ and $\boldsymbol{m}_{*}$ are aligned, that the ratio of mass flux to magnetic dipole flux is constant, and that the magnetic field is swept into a radial configuration between the Alfvén radius and the light cylinder. This normalization is $\sim 6$ times larger than deduced by TB 98 for a rotator with $\boldsymbol{m}_{*}$ inclined by $45^{\circ}$ with respect to $\Omega$ : they considered the enhanced torque resulting from the sweeping out of magnetic field lines but not the angular momentum of the outflow itself. ${ }^{8}$

The dipole magnetic field inferred from $P$ and $\dot{P}$ depends on the persistent wind luminosity. Normalizing $L_{\mathrm{A}}$ to the persistent X-ray luminosity, $L_{\mathrm{A}}=L_{\mathrm{A} 35} \times 10^{35} \mathrm{ergs} \mathrm{s}^{-1}$, one finds for SGR $1900+14$

$$
\begin{aligned}
B_{*}= & \times 10^{14} L_{\mathrm{A} 35}^{-1 / 2}\left(\frac{\Lambda}{2 / 3}\right)^{-1} I_{45} \\
& \times\left(\frac{\dot{P}}{6 \times 10^{-11}}\right)\left(\frac{P}{5.16 \mathrm{~s}}\right)^{-1} \mathrm{G} .
\end{aligned}
$$

A very strong magnetic field is needed to channel the flux of Alfvén waves and particles in corotation with the star out to a large radius. This extended "lever arm" enhances the magnetic braking torque for a given wind luminosity.

The surface dipole field of SGR $1900+14$ is inferred to be less than $B_{\mathrm{QED}}=4.4 \times 10^{13} \mathrm{G}$ only if $L_{\mathrm{A}}>10^{37} \mathrm{ergs} \mathrm{s}^{-1}$. That is, the wind must be $\sim 30-100$ times more luminous than the time-averaged X-ray output of the SGR in either quiescent or bursting modes. Such a large wind luminosity may conflict with observational bounds on the quiescent radio emission of SGR 1900+14 (Vasisht et al. 1994; Frail, Kulkarni, \& Bloom 1999). From these considerations alone (which do not involve the additional strong constraints from bursting activity) we find it difficult to reconcile the observed spin-down rate of SGR $1900+14$ with dipole fields typical of ordinary radio pulsars (as suggested recently by Marsden, Rothschild, \& Lingenfelter 1999).

Note also that the synchrotron nebula surrounding SGR 1806-20 (Kulkarni \& Frail 1993), thought until recently to emanate from the SGR itself and to require a particle source of luminosity $\sim 10^{37} \mathrm{ergs} \mathrm{s}^{-1}$ (TD96), appears instead to be

\footnotetext{
${ }^{8}$ Harding, Contopoulos, \& Kazanas (1999) have calculated this coefficient using an independent method and find $\Lambda=\sqrt{2} / 3$.
} 
TABLE 1

SGRs and AXPs with Measured SpIN-DOwn Rates and Associations WITH SUPERNOVA REMNANTS

\begin{tabular}{|c|c|c|c|c|c|}
\hline Source & $\begin{array}{l}\text { Period } \\
\text { (s) }\end{array}$ & $\begin{array}{l}\text { Period Derivative } \\
\qquad\left(\mathrm{s} \mathrm{s}^{-1}\right)\end{array}$ & $\begin{array}{l}t_{\mathrm{MDR}} \\
(\mathrm{yr})\end{array}$ & $\begin{array}{l}t_{\mathrm{SNR}} \\
(\mathrm{yr})\end{array}$ & $t_{\mathrm{MDR}} / t_{\mathrm{SNR}}$ \\
\hline SGR $1806-20^{\mathrm{a}}$ & 7.47 & $8.3 \times 10^{-11}$ & 1430 & $\sim 10^{4}$ & $\sim 0.1$ \\
\hline SGR $1900+14^{\mathrm{b}}$. & 5.16 & $6.1 \times 10^{-11}$ & 1340 & $\sim 10^{4}$ & $\sim 0.1$ \\
\hline AXP 1 E $2259+586^{\circ} \ldots \ldots$ & 6.98 & $5.0 \times 10^{-13}$ & 220000 & $\sim 13000$ & $\sim 10$ \\
\hline AXP 1 E $1841-045^{\mathrm{d}} \ldots \ldots$ & 11.8 & $4.13 \times 10^{-11}$ & 4570 & $\sim 2000$ & $\sim 2.3$ \\
\hline
\end{tabular}

${ }^{\text {a }}$ Kouveliotou et al. 1998.

b Woods et al. 1999b and references therein.

c Mereghetti, Israel, \& Stella 1998 and references therein; Wang et al. 1992.

d Vasisht \& Gotthelf 1997; Gotthelf \& Vasisht 1997; Gotthelf et al. 1999.

associated with a nearby luminous blue variable star discovered by van Kerkwijk et al. (1995). The new IPN localization of the SGR source (Hurley et al. 1999c) is displaced by $12^{\prime \prime}$ from the peak of the radio emission. There is no detected peak in radio emission at the revised location. Since the two SGRs have nearly identical $\dot{P} / P$, we estimate a dipole field $B_{*}=3 \times 10^{14} L_{\mathrm{A} 35}^{-1 / 2}$ G for SGR $1806-20$.

During episodes of wind-aided spin-down, the period grows exponentially:

$$
P(t)=\mathscr{P} \exp \left(t / \tau_{\mathrm{w}}\right),
$$

if the luminosity $L_{\mathrm{A}}$ in outflowing Alfvén waves and relativistic particles remains constant. In this equation, $\tau_{\mathrm{w}} \equiv$ $P / \dot{P}=I_{*} c^{3 / 2} /\left(\Lambda B_{*} R_{*}^{3} L_{\mathrm{A}}^{1 / 2}\right)$ is a characteristic braking time and $\mathscr{P}$ is the rotation period at the onset of wind-aided spin-down. If $L_{\mathrm{A}}$ has remained unchanged over the lifetime of the star, then $\mathscr{P}$ would be set by the condition that the Alfvén radius sit inside the light cylinder, $\mathscr{P}=$ $2 \pi\left(B_{*}^{2} R_{*}^{6} / c^{3} L_{\mathrm{A}}\right)^{1 / 4}=1.9 L_{\mathrm{A} 35}^{-1 / 4}\left(B_{* 14} / 3\right)^{1 / 2}$ s (see eq. [2]). (Here $B_{*}=10^{14} B_{* 14} \mathrm{G}$ is the polar magnetic field.)

The narrow distribution of spin periods in the SGR/AXP sources $(P=5-12 \mathrm{~s})$ would be hard to explain if every source underwent this kind of extended exponential spindown, but the possibility cannot be ruled out in any one source. The total age of such a source would be

$$
t=(P / \dot{P}) \ln (P / \mathscr{P})+t(\mathscr{P}),
$$

where $t(\mathscr{P})$ is the time required to spin down to period $\mathscr{P}$. Notice that $\dot{P} \propto P$ at constant $L_{\mathrm{A}}$, as compared with $\dot{P} \propto$ $P^{-1}$ in the case of magnetic dipole radiation (MDR). The net result is to lengthen the spin-down age deduced from a given set of $P$ and $\dot{P}$, relative to the usual estimate $t_{\mathrm{MDR}} \equiv$ $P / 2 \dot{P}$ employed for radio pulsars. Note also that $P / \dot{P}$ remains constant throughout episodes of wind-aided spindown.

Applying these results to SGR $1900+14$ (eq. [17]), we would infer that wind-aided spin-down has been operating for $(P / \dot{P}) \ln (P / \mathscr{P})=2700 \mathrm{yr}$ (assuming a steady wind of luminosity $\left.L_{\mathrm{A} 35}=1\right)$. Its total age, including the age $t(\mathscr{P})$ at the onset of wind-aided braking, would be $2700+1300=4000$ yr. (This number only increases to $5600 \mathrm{yr}$ if $L_{\mathrm{A}}$ increases to $10^{36} \mathrm{ergs} \mathrm{s}^{-1}$.) This age remains uncomfortably short to allow a physical association with SNR G42.8 + 0.6: it would imply a transverse recoil velocity $V_{\perp} \approx 0.03(D / 7 \mathrm{kpc}) c$ (eq. [13]).

The age of SGR $1900+14$ can be much longer, and $V_{\perp}$ much smaller, if the accelerated spin-down we now observe occurs only intermittently (eq. [14]). In the magnetar model, it is plausible that small-scale seismic activity and Alfvéndriven winds are only vigorous during transient episodes, which overlap periods of bursting activity.

\subsection{Connection with Anomalous $X$-Ray Pulsars}

If each magnetar undergoes accelerated spin-down only for a fraction $\epsilon_{\text {active }} \sim P / \dot{P} t_{\mathrm{SNR}} \sim 0.25$ of its life (eq. [14]), then the observed SGRs should be outnumbered some $\epsilon_{\text {active }}^{-1} \sim 4$ times by inactive sources that spin down at a rate $\dot{P} \leq P / 2 t_{\mathrm{SNR}}$.

The AXPs have been identified as such inactive SGRs (Duncan \& Thompson 1996; TD96; Vasisht \& Gotthelf 1997; Kouveliotou et al. 1998). Although harder to find because they do not emit bright bursts, six AXPs are already known in our Galaxy, as compared with three Galactic SGRs. Table 1 summarizes the spin behavior and age estimates of the two AXP sources that are presently associated with supernova remnants $(1 \mathrm{E} 2259+586$ and $1 \mathrm{E}$ $1841-045)$. Their characteristic ages are larger than those of SGR $1900+14$ and SGR 1806-20.

The characteristic age of 1E $2259+586$ also appears to be much longer, by about an order of magnitude, than the age of the associated SNR CTB 109. From Wang et al. (1992),

$$
t_{\mathrm{SNR}}=13,000\left(\frac{E_{\mathrm{SN}}}{0.4 \times 10^{51} \mathrm{ergs}}\right)^{-1 / 2}\left(\frac{n}{0.13 \mathrm{~cm}^{-3}}\right)^{1 / 2} \mathrm{yr},
$$

where $E_{\mathrm{SN}}$ is the supernova energy and $n$ is the interstellar medium particle density into which the remnant has expanded. Such a large characteristic age has a few possible explanations in the magnetar model. First, the source may previously have undergone a period of wind-aided spindown that increased its period to $\sim 4$ times the value that it would have reached by magnetic dipole braking alone. Indeed, there is marginal evidence for an extended X-ray halo surrounding the source, suggesting recent output of energetic particles (Rho \& Petre 1997).

Second, the long characteristic age of 1E $2259+586$ could be caused by significant decay of the dipole field (TD93, §§ 14.3 and 15.2). A third mechanism for torque reduction involves the alignment of a vacuum magnetic dipole with the axis of rotation (Davis \& Goldstein 1970; Michel \& Goldwire 1970). In this case, episodes of seismic activity can increase the spin-down torque in aligned rotators both by driving the conduction current above the displacement current in the outer magnetosphere and by carrying off angular momentum in particles and waves. 
Indeed, the outer boundary of the rigidly corotating magnetosphere, calculated by Melatos (1997) to lie at a radius ${ }^{9}$ $R_{\mathrm{mag}} / R_{*}=1 \times 10^{3} \gamma^{-1 / 5}\left(B_{*} / 10^{14} \mathrm{G}\right)^{2 / 5}$, is contained well inside the speed of light cylinder, $R_{\mathrm{lc}} / R_{*}=3 \times 10^{4}(P / 6 \mathrm{~s})$. If this last mechanism applies to the SGRs, then their accelerated torque could be comparable to that of an orthogonal vacuum rotator, so that the inferred polar field is as high as $\sim 1 \times 10^{15} \mathrm{G}$. Here $\gamma$ is the bulk Lorentz factor of the streaming charges. There may be some tendency toward an initial alignment of $\boldsymbol{m}_{*}$ and $\Omega$ in rapidly rotating neutron stars that support a large scale $\alpha-\Omega$ dynamo. However, rapid magnetic field decay will generically force $\boldsymbol{m}_{*}$ out of alignment with $\Omega$ and the principal axes of the star.

The remarkable AXP 1841-045 discovered by Vasisht \& Gotthelf (1997) is only $\sim 2000 \mathrm{yr}$ old, as inferred from the age of the counterpart supernova remnant (Gotthelf \& Vasisht 1997). The ratio $t_{\mathrm{MDR}} / t_{\mathrm{SNR}}$ is consistent with unity, in contrast with all other magnetar candidates that have measured spin-down and are associated with supernova remnants (Table 1). Of these sources, AXP $1841-045$ is also unique in failing to show measurable variations in its spindown rate, X-ray luminosity, or X-ray pulse shape over 10 yr (Gotthelf, Vasisht, \& Dotani 1999), nor has it emitted any $\mathrm{X}$-ray bursts or evinced any evidence for a particle outflow through a radio synchrotron halo. These facts reinforce the hypothesis that departures from simple magnetic dipole braking are correlated with internal activity in a magnetar and suggest that inactive phases can occur early in the life of a magnetar. In particular, the crust will be deformed plastically by magnetic stresses when $B^{2} / 4 \pi$ exceeds the shear modulus (TD96), corresponding to $B>6 \times 10^{15} \mathrm{G}$ in the deep crust.

\subsection{Free Precession in SGRs and AXPs}

Magnetic stresses will distort the shape of a magnetar, thereby allowing for the possibility of long-period precession (Melatos 1999). The internal magnetic field generated by a post-collapse $\alpha-\Omega$ dynamo is probably dominated by a toroidal component (DT92; TD93). A field stronger than $\sim 100 B_{\text {QED }}$ is transported through the core and deep crust of the neutron star on a timescale short enough for SGR activity (TD96). Such a magnetar is initially prolate, with quadrupole moment $\epsilon=1 \times 10^{-5}\left(B_{\mathrm{in}} / 100 B_{\mathrm{QED}}\right)^{2}$ (Bonazzola \& Gourgoulhon 1996). Rapid field decay may cause the magnetic moment $\boldsymbol{m}_{*}$ to rotate away from the long principal axis $\hat{z}$ of the star, irrespective of any initial tendency for these two axes to align. The distortion of the rotating figure of the star induced by the rigidity of the crust can be neglected when calculating the spin evolution of the star, as long as $B>10^{12}(P / 1 \mathrm{~s})^{-1} \mathrm{G}$ (Goldreich 1970).

This hydromagnetic distortion gives rise to free precession on a timescale

$$
\tau_{\mathrm{pr}}=\frac{2 \pi}{\epsilon \Omega}=2 \times 10^{-2}\left(\frac{B_{\mathrm{in}}}{100 B_{\mathrm{QED}}}\right)^{-2}\left(\frac{P}{6 \mathrm{~s}}\right) \mathrm{yr} .
$$

Even when the magnetosphere is loaded with plasma, the spin-down torque will depend on the angle between $\boldsymbol{m}_{*}$ and the angular velocity $\Omega$. Free precession modulates this angle when $\boldsymbol{m}_{*}$ is canted with respect to the long principal axis $\hat{z}$ and so induces a periodic variation in the spin-down torque.

\footnotetext{
${ }^{9}$ When the displacement current dominates the conduction current.
}

Observation of free precession in an SGR or AXP source would provide a direct measure of its total magnetic energy.

An argument of Shaham (1977) suggests that long-period precession is possible only if the crustal neutron superfluid is weakly pinned. A precession period $\tau_{\mathrm{pr}}$ requires that the moment of inertia of the pinned crustal superfluid does not exceed $I_{\text {pinned }} / I_{\mathrm{NS}} \sim P / \tau_{\mathrm{pr}}=3 \times 10^{-7}\left(\tau_{\mathrm{pr}} / 1 \mathrm{yr}\right)^{-1}(P / 8 \mathrm{~s})$ for a precession period $\tau_{\mathrm{pr}}$, several orders of magnitude smaller than is inferred for young, glitching pulsars.

In the realistic case of a plasma-loaded magnetosphere, the rate at which free precession is excited or damped by electromagnetic and particle torques is, unfortunately, not yet known. An internal excitation mechanism, which may be particularly effective in an active SGR, involves rapid transport of the field in short, intense bursts. This is a likely consequence of energetic flares like the March 5 or August 27 events, which probably have occurred $\sim 10^{2}$ times over the lifetimes of these sources. If the principal axes of the star are rearranged on a timescale less than $\tau_{\mathrm{pr}}$, then $\Omega$ will not have time to realign with the principal axes and precession is excited. Only if the magnetic field is transported on a timescale longer than $\tau_{\mathrm{pr}}$ will $\Omega$ adiabatically track the principal axes.

Melatos (1999) made the related suggestion that forced radiative precession in a magnetar drives the bumpy spindown of the AXP sources 1E 2259+586 and 1E 1048-593 on a timescale of years. When $\boldsymbol{m}_{*}$ is not aligned with $\Omega$, the asymmetric inertia of the corotating magnetic field induces a torque along $\Omega \times \boldsymbol{m}_{*}$ (Davis \& Goldstein 1970). This near-field torque acts on a timescale $\tau_{\mathrm{nf}}$ that is $(\Omega R / c)$ times the electromagnetic braking time:

$$
\tau_{\mathrm{nf}} \simeq 0.3\left(\frac{B_{*}}{10 B_{\mathrm{QED}}}\right)^{-2}\left(\frac{P}{6 \mathrm{~s}}\right) \mathrm{yr} .
$$

In particular, Melatos (1999) considers the case in which $\tau_{\mathrm{nf}} \sim \tau_{\mathrm{pr}}$, and the near-field torque drives an anharmonic wobble of the neutron star. However, inspection of equations (21) and (22) suggests instead that $\tau_{\mathrm{pr}} \ll \tau_{\mathrm{nf}}$ whenever the magnetic energy is dominated by an internal (toroidal) component. In this case, the effect of the near-field torque is smaller (Goldreich 1970). The main consequence of the asymmetric inertia of the corotating fields is to shift slightly the principal axes of the star. The model has the virtue of making clear predictions of the future rotational evolution of the AXPs, which will be tested in coming years.

\section{CHANGES IN THE PERSISTENT X-RAY FLUX AND LIGHT CURVE}

The persistent X-ray light curve of SGR $1900+14$ measured following the August 27 event (Kouveliotou et al. 1999; Murakami et al. 1999) appears dramatically different from the pulse profile measured earlier: indeed, the profile measured following the burst activity of 1998 May/June (Kouveliotou et al. 1999) is identical to that measured in 1998 April (Hurley et al. 1999b) and 1996 September (Marsden et al. 1999). Not only did the pulse-averaged luminosity increase by a factor of 2.3 between the 1998 April 30 and 1998 September 17/18 ASCA observations (Hurley et al. 1999b; Murakami et al. 1999), but the light curve also simplified into a single prominent pulse, from a multipulsed profile before the August 27 flare. The brighter, simplified light curve is suggestive of enhanced dissipation in the active region of the outburst (Kouveliotou et al. 
1999). We now discuss the implications of this observation for the dissipative mechanism that generates the persistent $\mathrm{X}$-rays, taking into account the additional constraints provided by the period history of SGR $1900+14$.

\subsection{Persistent Magnetospheric Currents}

The X-ray output of a magnetar can be divided into two components (TD96): thermal conduction to the surface, driven by heating in the core and inner crust; and external Comptonization and particle bombardment powered by persistent seismic activity in the star. Both mechanisms naturally generate $\sim 10^{35} \mathrm{ergs} \mathrm{s}^{-1}$ in continuous output. The appearance of a thermal pulse at the surface of the neutron star will be delayed with respect to a deep fracture or plastic rearrangement of the neutron star crust, by the thermal conduction time of $\sim 1 \mathrm{yr}$ (e.g., Van Riper, Epstein, \& Miller 1991). By contrast, external heating will vary simultaneously with seismic activity in the star. We have previously argued that if $1 \mathrm{E} 2259+586$ is a magnetar, then the coordinated rise and fall of its two X-ray pulses (as observed by Ginga; Iwasawa, Koyama, \& Halpern 1992) requires the thermal component of the X-ray emission to be powered, in part, by particle bombardment of two connected magnetic poles (TD96, § 4.2).

Neither internal heating nor variability in the rate of persistent seismic activity appears able to provide a consistent explanation for the variable light curve of SGR $1900+14$. Deposition of $\sim 10^{44}$ ergs of thermal energy in the deep crust, of which a fraction $1-\epsilon$ is lost to neutrino radiation, will lead to an increased surface X-ray output of $\sim 3 \times 10^{35}(\epsilon / 0.1)$ ergs s $^{-1}$. If, in addition, the heat deposited per unit mass is constant with depth $z$ in the crust, then the heat per unit area scales as $\sim z^{4}$, whereas the thermal conduction time varies weakly with $z$ at densities above neutron drip (Van Riper et al. 1991). The outward heat flux should, as a result, grow monotonically. This conflicts with the appearance of the new pulse profile of SGR $1900+14$ no later than one day after the August 27 event. By the same token, a significant increase in persistent seismic activity, at the rate needed to power the increased persistent luminosity $L_{\mathrm{X}} \sim 1.5 \times 10^{35}(\mathrm{D} / 7 \mathrm{kpc})^{2} \mathrm{ergs} \mathrm{s}^{-1}$ (Murakami et al. 1999), would induce a measurable change in the spin-down rate that was not observed.

The observations require instead a steady particle source that is confined to the inner magnetosphere. A large-scale deformation of the crust of the neutron star, which likely occurred during the August 27 outburst, must involve a horizontal twisting motion ( $\$ 3$ ). If this motion were driven by internal magnetic stresses, ${ }^{10}$ then the external magnetic field lines connected to the rotating patch would be twisted with respect to their opposite footpoints (which we assume to remain fixed in position). We suppose that the twist angle decreases smoothly from a value $\theta_{\max }$ at the center of the patch to its boundary at radius $a$. This means that a component of the twist will remain even after magnetic recon-

\footnotetext{
${ }^{10} \mathrm{~A}$ sudden unwinding of an external magnetic field could release enough energy to power the March 5 (or August 27) event, but it was argued in TD95 that the timescale $\sim R_{*} / c \sim 10^{-4} \mathrm{~s}$ would be far too short to explain the width of the initial $\sim 0.2 \mathrm{~s}$ hard spike. A pulse broadened by a heavy matter loading would suffer strong adiabatic losses and carry a much greater kinetic energy than is observed in $\gamma$-rays. Shearing of the external magnetic field requires internal motions that will, in themselves, trigger a large outburst by fracturing the crust.
}

nection eliminates any tangential discontinuities in the external magnetic field resulting from the motion. The current carried by the twisted bundle of magnetic field is

$$
I \simeq \frac{\theta_{\max } \Phi c}{8 \pi L},
$$

where $\Phi=\pi a^{2} B_{*}$ is the magnetic flux carried by the bundle and $L$ is its length.

The surface of an AXP or SGR is hot enough $(T \sim 0.5$ $\mathrm{keV}$ ) to feed this current via thermionic emission of $Z<12$ ions from one end of the flux bundle and electrons from the other end. In magnetic fields stronger than $Z^{3} \alpha_{\mathrm{em}}^{2} B_{\mathrm{QED}}=4$ $\times 10^{13}(Z / 26)^{3} \mathrm{G}$, even iron is able to form long molecular chains. The cohesion energy per atom is

$$
\begin{aligned}
\frac{\Delta E}{Z^{3}(13.6 \mathrm{eV})}= & 1.52\left(\frac{B}{Z^{3} \alpha_{\mathrm{em}}^{2} B_{\mathrm{QED}}}\right)^{0.37} \\
& -\frac{7}{24}\left[\ln \left(\frac{B}{Z^{3} \alpha_{\mathrm{em}}^{2} B_{\mathrm{QED}}}\right)\right]^{2} .
\end{aligned}
$$

In this expression, the first term is the binding energy per atom in the chain (Neuhauser, Koonin, \& Langanke 1987; Lai, Salpeter, \& Shapiro 1992), from which we subtract the binding energy of an isolated atom (Lieb, Solovej, \& Yngvason 1992). Thermionic emission of ions is effective above a surface temperature

$$
T_{\text {thermionic }} \simeq \frac{\Delta E}{30} .
$$

Substituting $B=10 B_{\mathrm{QED}}=4.4 \times 10^{14} \mathrm{G}$, one finds that $T_{\text {thermionic }}$ remains well below $0.5 \mathrm{keV}$ for $Z<12$ but grows rapidly at higher $Z$. Thus, the surface of a magnetar should be an effective thermionic emitter for a wide range of surface compositions.

We can now estimate the energy dissipated by the current flow. Only a small parallel electric field $E=-(A / Z) m_{p}$ $g \cdot \hat{\boldsymbol{B}} / e$ is needed to cancel the gravitational force on the ions of charge $Z$ and mass $A$. The ions can be lifted off the surface of the neutron star by their thermal motion, whereas the counterstreaming electrons are electrostatically accelerated to bulk relativistic speed. This work done on the electrons is eventually released in the form of Comptonized thermal X-ray photons, with a luminosity

$$
\begin{aligned}
L_{\mathrm{X}}=\left(\frac{A}{Z}\right) \frac{I m_{p} \phi}{e}=3 & \times 10^{35} \frac{\theta_{\max } A}{Z}\left(\frac{B_{*}}{10 B_{\mathrm{QED}}}\right) \\
& \times\left(\frac{L}{R_{*}}\right)^{-1}\left(\frac{a}{0.5 \mathrm{~km}}\right)^{2} \mathrm{ergs} \mathrm{s}^{-1} .
\end{aligned}
$$

Here $\phi \simeq g_{*} R_{*}=G M_{*} / R_{*}$ is the gravitational potential that the ions have to climb along the tube, and we assume $M_{*}=1.4 M_{\odot}, R_{*}=10 \mathrm{~km}$.

Note that the particle flow estimated here is large enough to break up heavy nuclei even where the outflowing current has a positive sign: electrons returning from the opposite magnetic footpoint are energetic enough for electroninduced spallation to be effective (e.g., Schaeffer, Reeves, \& Orland 1982).

On what timescale will this twist decay? Each charge accumulates a potential energy $A m_{p} g z$ a height $z$ above the surface of the neutron star. Equating this energy with the electrostatic energy released along the magnetic field, one 
requires a longitudinal electric field $E=A m_{p} g / Z e$. The corresponding electrical conductivity is

$$
\sigma=\frac{I}{\pi a^{2} E}=\left(\frac{Z \theta_{\max }}{8 \pi A}\right) \frac{e B c}{m_{p} g_{*} L},
$$

and the ohmic decay time is

$$
\begin{aligned}
t_{\mathrm{ohmic}} & =\frac{4 \pi \sigma L^{2}}{c^{2}}=\left(\frac{Z \theta_{\max }}{2 A}\right) \frac{e B_{*} L}{m_{p} g_{*} c} \\
& =300\left(\frac{Z \theta_{\max }}{A}\right)\left(\frac{B_{*}}{10 B_{\mathrm{QED}}}\right)\left(\frac{L}{10 \mathrm{~km}}\right) \mathrm{yr} .
\end{aligned}
$$

This timescale agrees with that obtained by dividing the persistent luminosity $L_{\mathbf{X}}$ into the available energy of the twisted magnetic field. (Further twisting of the field lines would prolong or shorten the lifetime of the current flow.) Since $t_{\text {ohmic }}$ is smaller than the $\sim 10^{4} \mathrm{yr}$ age of an SGR or AXP, this provides a mechanism that correlates hard persistent X-ray spectra $\left(\alpha_{X} \sim 2\right.$ for SGR $1806-20$ and SGR $1900+14)$ with bursting activity.

A static twist in the surface magnetic field will not produce a measurable increase in the torque because the current flow is contained well inside the Alfvén radius (eq. [15]). The particles that carry the current lose their energy to Compton scattering and surface impact on a timescale $\sim R_{*} / c$ or shorter. By contrast, a persistent flux of lowamplitude Alfvén waves into the magnetosphere causes the wave intensity to build up, until the wave luminosity transported beyond the Alfvén radius balances the continuous output of the neutron star (TB98). Thus, the particle flow induced by a localized twist in the magnetic field lines supplements the particle output associated with persistent seismic activity occurring over the larger volume of the star.

\subsection{Evidence against Persistent Accretion}

Direct evidence that the persistent X-ray output of SGR $1900+14$ is not powered by accretion comes from measurements one day after the August 27 outburst (Kouveliotou et al. 1999). The increase in persistent $L_{\mathrm{X}}$ is not consistent with a constant spin-down torque, unless there was a substantial change in the angular pattern of the emergent X-ray flux following the burst. In addition, the radiative momentum deposited by that outburst on a surrounding accretion disk would more than suffice to expel the disk material, out to a considerable distance from the neutron star. In such a circumstance, the time to reestablish the accretion flow onto the neutron star, via inward viscous diffusion from the inner boundary $R_{\text {in }}$ of the remnant disk, would greatly exceed 1 day. ${ }^{11}$ Let us consider this point in more detail.

The accretion rate (assumed steady and independent of radius before the outburst) is related to the surface mass density $\Sigma(R)$ of the hypothetical disk via

$$
\dot{M}=\frac{2 \pi R^{2} \Sigma(R)}{t_{\mathrm{visc}}(R)} .
$$

\footnotetext{
${ }^{11}$ This estimate of the viscous timescale is conservative for two reasons. First, if the binding energy of the disk material were balanced with the incident radiative energy, the inner boundary of the remnant disk would lie at even larger radius. Second, the central X-ray source may puff up the disk, which increases $\tau_{\text {visc }}$ (eq. [31]).
}

The viscous timescale is, as usual,

$$
t_{\text {visc }}(R) \simeq \alpha_{\mathrm{SS}}^{-1}\left[\frac{H(R)}{R}\right]^{-2}\left(\frac{R^{3}}{G M_{*}}\right)^{1 / 2},
$$

where $H(R)$ is the half-thickness of the disk at radius $R$ and $\alpha_{\mathrm{SS}}<1$ is the viscosity coefficient (Shakura \& Sunyaev 1973). Balancing the radiative momentum incident on a solid angle $\sim 2 \pi(2 H / R)$ against the momentum $\sim \pi \Sigma(R)$ $R^{2}\left(2 G M_{*} / R\right)^{1 / 2}$ of the disk material moving at the escape speed and equating the persistent X-ray luminosity $L_{\mathrm{X}}$ with $G M_{*} \dot{M} / R_{*}$, one finds

$$
t_{\mathrm{visc}}=\frac{E_{\mathrm{Aug} 27}}{L_{\mathrm{X}}}\left(\frac{2 G M_{*}}{R_{*} c^{2}}\right)^{1 / 2}\left(\frac{R_{\mathrm{in}}}{R_{*}}\right)^{1 / 2}\left[\frac{H\left(R_{\mathrm{in}}\right)}{R_{\mathrm{in}}}\right] .
$$

The most important factor in this expression is the ratio of burst energy to persistent X-ray luminosity, $E_{\mathrm{Aug} 27} / L_{\mathrm{X}}=$ $30\left(E_{\text {Aug2 } 7} / 10^{44} \mathrm{ergs}\right)\left(L_{\mathrm{X}} / 10^{35} \mathrm{ergs} \mathrm{s}^{-1}\right)$ yr. The timescale is long as a result of the enormous energy of the August 27 flare and the relatively weak persistent X-ray flux preceding it. It is interesting to compare with type II X-ray bursts from the Rapid Burster and GRO J1744-28, which are observed to be followed by dips in the persistent emission (Lubin et al. 1992; Kommers et al. 1997). These bursts, which certainly are powered by accretion, involve energies $\sim 10^{4}$ times smaller and a persistent source luminosity that is $10^{2}-10^{3}$ times higher. Indeed, the dips in the persistent emission following the type II bursts last for only 100-200 s, consistent with the above formula.

Now let us evaluate equation (31) in more detail. At a fixed $\dot{M}$, the surface mass density of the disk increases with decreasing $\alpha_{\mathrm{SS}}$, and so a conservative upper bound on $t_{\mathrm{visc}}$ is obtained by choosing $\alpha_{\text {ss }}$ to be small. (Note that eq. [31] depends implicitly on $\alpha_{\mathrm{SS}}$ only through the factor of $R_{\mathrm{in}}^{1 / 2} \propto$ $\alpha_{S S}^{1 / 2}$.) For the observed parameters $E_{\text {Aug27 }} \simeq 10^{44}$ ergs (Mazets et al. 1999) and $L_{\mathrm{X}}=10^{35} \mathrm{ergs} \mathrm{s}^{-1}$ (before the August 27 outburst; Hurley et al. 1999b), one finds $R_{\mathrm{in}}=1$ $\times 10^{10} \mathrm{~cm}$ when $\alpha_{\mathrm{SS}}=0.01$. The corresponding thickness of the gas pressure-dominated disk is (Novikov \& Thorne 1973) $H\left(R_{\text {in }}\right) / R_{\text {in }} \simeq 5 \times 10^{-3}$. The timescale over which the persistent X-ray flux would be reestablished is extremely long, $t_{\mathrm{visc}} \simeq 10 \mathrm{yr}$.

One final note on disk accretion. There is no observational evidence for a binary companion to any SGR or AXP (Kouveliotou 1999). Because of its large recoil velocity (eq. [13]), SGR 1900+14 almost certainly could not remain bound in a binary system. A similar argument applies to the other giant flare source, SGR 0526-66 (DT92). Thus, any accretion onto SGR $1900+14$ would have to come from a fossil disk. To remain bound, the initial radius of such a disk must be less than $G M_{*} / V_{\mathrm{rec}}^{2} \sim 10^{4} \mathrm{~km}$, for stellar recoil velocity $V_{\text {rec }} \sim(3 / 2)^{1 / 2} V_{\perp}$ (eq. [13]). The behavior of a passively spreading remnant disk appears inconsistent with the measured spin evolution of the AXP and SGR sources (Li 1999).

A trigger involving sudden accretion of an unbound planetesimal (Colgate \& Petschek 1981) is not consistent with the lognormal distribution of waiting periods between bursts (Hurley et al. 1994) in SGR 1806-20. An internal energy source is also indicated by the power-law distribution of burst energies, with index $d N / d E \sim E^{-1.6}$ similar to the Gutenburg-Richter law for earthquakes (Cheng et al. 1996). In addition, the mass of the accreted planetesimals must exceed $\sim 1 / 30$ times the mass of Earth's Moon in the 
case of the March 5 and August 27 events. It is very difficult to understand how the accretion of a baryon-rich object could induce a fireball as clean as the initial spike of these giant flares (TD95, § 7.3.1). When $B_{*} \ll 10^{14} \mathrm{G}$, only a tiny fraction $\left(B_{*} / B_{E}\right)$ of the hydrostatic energy released would be converted to magnetic energy; here $B_{E} \sim 10^{14} \mathrm{G}$ is the minimum field needed to directly power the outburst.

\section{CONCLUSIONS}

The observation (Paper I) of a rapid spin-down associated with the August 27 event, $\Delta P / P=1 \times 10^{-4}$, provides an important clue to the nature of SGR $1900+14$. We have described two mechanisms that could induce such a rapid loss of angular momentum from the crust and charged interior of the star. The torque imparted by a relativistic outflow during the August 27 event is proportional to $B_{*}$ but falls short by an order of magnitude even if $B_{*}$ $\sim 10 B_{\text {OED }}=4.4 \times 10^{14}$ G. Only if SGR $1900+14$ released an additional $\sim 10^{44}$ ergs for an extended period of $\sim 10^{4} \mathrm{~s}$ immediately following the August 27 outburst would the loss of angular momentum be sufficient. [The integrated torque increases with the duration $\Delta t$ of the outflow as $(\Delta t)^{1 / 2}$; eq. (4).]

The alternative model, which we favor, involves a glitch driven by the violent disruption of the August 27 event. The unpinned neutron superfluid will absorb angular momentum if it starts out spinning more slowly than the rest of the star, the opposite of the situation encountered in glitching radio pulsars. We have argued that a slowly spinning neutron superfluid is the natural consequence of magnetic stresses acting on the neutron star crust. A gradual, plastic deformation of the crust during the years preceding the recent onset of bursting activity in SGR $1900+14$ would move the superfluid out of corotation with the rest of the star and slow its rotation. The magnitude of the August 27 glitch can be crudely estimated by scaling to the largest glitches of young, active pulsars with similar spin-down ages and internal temperatures. Depending on the object considered, one deduces $|\Delta P| / P \sim 10^{-5}$ to $10^{-4}$.

This model for the August 27 period increment has interesting implications for the longer term spin-down history of the SGRs and AXPs. It suggests that these objects can potentially glitch, with or without associated bursts, and that $P$ will suddenly shift upward, rather than downward as in radio pulsar glitches. By the same token, an accelerated rate of plastic deformation within a patch of the neutron star crust will force the superfluid farther out of corotation and induce a transient (but potentially resolvable) spin-up of the crust (TD96). The magnitude of such a "plastic spinup" event (eq. [12]) could approach that inferred for the August 27 event, but with the usual (negative) sign observed in radio pulsar glitches. Indeed, $R X T E$ spin measurements provide evidence for a rapid spin-up of the AXP source 1E $2259+586$ (Baykal \& Swank 1996), to the tune of $\Delta P /$ $P=-3 \times 10^{-5}$. Transient variations in the persistent X-ray flux of the AXP 1E 2259+586, which were not associated with any large outbursts, also require transient plastic deformations of the neutron star crust (TD96).

The rapid spin-down rate of SGR $1900+14$ during the past few years, $\dot{P}=6 \times 10^{-11} \mathrm{~s} \mathrm{~s}^{-1}$, indicates that this SGR is a transient phase of accelerated spin-down, with stronger braking torques than would be produced by simple magnetic dipole radiation (Kouveliotou et al. 1999). Such accelerated spin-down can be driven by magnetically induced seismic activity, with small-scale fractures powering a steady relativistic outflow of magnetic vibrations and particles. This outflow, when channeled by the dipole magnetic field, carries away the star's angular momentum. A very strong field, $B_{*} \gg B_{\mathrm{QED}}$, is required to give a sufficiently large "lever arm" to the outflow.

Further evidence for episodic accelerated spin-down comes from the two AXPs that are directly associated with supernova remnants: $1 \mathrm{E} 2259+586$ and $1 \mathrm{E}$ 1841-045 ( $\$$ 4.2). The characteristic ages $P / 2 \dot{P}$ of these stars are longer than the ages of the associated supernova remnant and also longer than the characteristic ages of the SGRs. This suggests that the AXPs are magnetars observed during phases of seismic inactivity.

The constancy of the long-term spin-down rate before and after the bursts and giant flare of 1998 (Woods et al. 1999c; Marsden et al. 1999; Paper I) gives evidence that the spin-down rate correlates only weakly with bursting activity. It is easy to understand why short, intense bursts are not effective at spinning down a magnetar: the Alfvén radius (the length of the "lever arm") decreases as the flux of Alfvén waves and particles increases.

A persistent output of waves and particles could be driven by the compressive mode of ambipolar diffusion in the liquid neutron star interior (TD96). As the magnetic field is forced through the crust, the Hall term in the electrical conductivity induces many frequent, small fractures $\left(\Delta E \sim 10^{35}\right.$ ergs). By contrast, large fractures of the crust are driven by shear stresses that involve the orthogonal (rotational) mode of ambipolar diffusion. The greater intermittency of bursting activity is a direct consequence of the dominance of the total burst fluence by the largest bursts (Cheng et al. 1996).

A twist in the exterior magnetic field induced by a largescale fracture of the crust will force a persistent thermionic current through the magnetosphere ( $\S 5$ ). The resulting steady output in particles would explain the factor of $\sim 2.3$ increase in the persistent X-ray flux of SGR $1900+14$ immediately following the August 27 event (Murakami et al. 1999) if $B_{*} \sim 10 B_{\mathrm{QED}}$ and the twist is through $\sim 1$ radian. In this model, the simplification of the light curve (into a single large pulse) is due to concentrated particle heating at the site of the August 27 event.

We conclude by emphasizing the diagnostic potential of coordinated measurements of spectrum, flux, bursting behavior, and period derivative. When considered together, they constrain not only the internal mechanism driving the accelerated spin-down of an SGR source but also the mechanism powering its persistent X-ray output. For example, an increase in surface X-ray flux will be delayed by $\sim 1 \mathrm{yr}$ with respect to an episode of deep heating (e.g., Van Riper et al. 1991), whereas a shearing and twisting of the external magnetic field of the neutron star will drive a simultaneous increase in the rate of external particle heating (TD96). The magnetar model offers a promising framework in which to interpret these observations.

We acknowledge support from NASA grant NAG 5-3100 and the Alfred P. Sloan foundation (C. T.), NASA grant NAG5-8381 and Texas Advanced Research Project grant ARP-028 (R. C. D.), the cooperative agreement NCC 8-65 (P. M. W.), and NASA grants NAG5-3674 and NAG5-7808 (J. v. P.). C. T. thanks A. Alpar and M. Ruderman for conversations. 


\section{REFERENCES}

Alpar, M. A., Anderson, P. W., Pines, D., \& Shaham, J. 1984, ApJ, 276, 325 Alpar, M. A., Chau, H. F., Cheng, K. S., \& Pines, D. 1996, ApJ, 459, 706 Baykal, A., \& Swank, J. H. 1996, ApJ, 460, 470

Blaes, O., Blandford, R., Goldreich, P., \& Madau, P. 1989, ApJ, 343, 829

Bonazzola, S., \& Gourgoulhon, E. 1996, A\&A, 312, 675

Cheng, B., Epstein, R. I., Guyer, R. A., \& Young, C. 1996, Nature, 382, 518

Cline, T., et al. 1982, ApJ, 255, L45

Colgate, S. A., \& Petchek, A. G. 1981, ApJ, 248, 771

Davis, L., \& Goldstein, M. 1970, ApJ, 159, L81

Duncan, R. C., \& Thompson, C. 1992, ApJ, 392, L9 (DT92)

. 1996, in AIP Conf. Proc. 366, High-Velocity Neutron Stars and Gamma Ray Bursts, ed. R. E. Rothschild \& R. E. Lingenfelter (Woodbury: AIP), 111

Feroci, M., Frontera, F., Costa, E., Amati, L., Tavani, M., Rapisarda, M., \& Orlandini, M. 1999, ApJ, 515, L9

Frail, D. A., Kulkarni, S. R., \& Bloom, J. S. 1999, Nature, 398, 127

Gogus, E., Woods, P. M., Kouveliotou, C., van Paradijs, J., Briggs, M. S., Duncan, R. C., \& Thompson, C. 1999, ApJ, 526, L93 2000, ApJ, 532, L121

Goldreich, P. 1970, ApJ, 160, L11

Gotthelf, E. V., \& Vasisht, G. 1997, ApJ, 486, L133

Gotthelf, E. V., Vasisht, G., \& Dotani, T. 1999, ApJ, 522, L49

Harding, A. K., Contopoulos, I., \& Kazanas, D. 1999, ApJ, 525, L125

Herold, H. 1979, Phys. Rev. D, 19, 2668

Heyl, J. S., \& Hernquist, L. 1999, MNRAS, 304, L37

Heyl, J. S., \& Kulkarni, S. R. 1998, ApJ, 506, L61

Hurley, K., et al. 1996, ApJ, 463, L13 1999a, Nature, 397, 41 - 1999b, ApJ, 510, L111

Hurley, K., Kouveliotou, C., Cline, T., Mazets, E. P., Golenetskii, S., Frederiks, D. D., \& van Paradijs, J. 1999c, ApJ, 523, L37

Hurley, K., Kouveliotou, C., Woods, P., Cline, T., Butterworth, P., Mazets, E., Golenetski, S., \& Frederics, D. 1999d, ApJ, 510, L107

Hurley, K., Kouveliotou, C., Woods, P., Mazets, E., Golenetskii, S., Frederiks, D. D., Cline, T., \& van Paradijs, J. 1999e, ApJ, 519, L143

Hurley, K., Sommer, M., Kouveliotou, C., Fishman, G., Meegan, C., Cline, T., Boer, M., \& Niel, M. 1994, ApJ, 431, L31

Inan, U. S., Lehtinen, N. G., Lev-Tov, S. J., Johnson, M. P., Bell, T. F., \& Hurley, K. 1999, Geophys. Res. Lett., 26, 3357

Iwasawa, K., Koyama, K., \& Halpern, J. P. 1992, PASJ, 44, 9

Kommers, J. M., Fox, D. W., Lewin, W. H. G., Rutledge, R. E., van Paradijs, J., \& Kouveliotou, C. 1997, ApJ, 482, L53

Kouveliotou, C. 1999, Proc. Nat1. Acad. Sci., 96, 5351

Kouveliotou, C., et al. 1998, Nature, 393, 235

. 1999, ApJ, 510, L115

Kulkarni, S., \& Frail, D. A. 1993, Nature, 365, 33

Kulkarni, S., Frail, D. A., Kassim, N. E., Murakami, T., \& Vasisht, G. 1994, Nature, 368, 129

Lai, D., Salpeter, E. E., \& Shapiro, S. L. 1992, Phys. Rev. A, 45, 4832

Li, X.-D. 1999, ApJ, 520, 271
Lieb, E. H., Solovej, J. P., \& Yngvason, J. 1992, Phys. Rev. Lett., 69, 749

Link, B., \& Epstein, R. I. 1996, ApJ, 457, 844

Link, B., Epstein, R. I., \& Baym, G. 1993, ApJ, 403, 285

Lubin, L. M., Lewin, W. H. G., Rutledge, R. E., van Paradijs, J., van der

Klis, M., \& Stella, L. 1992, MNRAS, 258, 759

Marsden, D., Rothschild, R. E., \& Lingenfelter, R. E. 1999, ApJ, 520, L107

Mazets, E. P., Cline, T., Aptekar, R. L., Butterworth, P., Frederiks, D. D., Golenetskii, S. V., Il'inskii, V. N., \& Pal'shin, V. D. 1999, Astron. Lett., 25,635

Mazets, E. P., Golenetskii, S. V., Ilinskii, V. N., Aptekar, R. L., \& Guryan, Iu. A. 1979, Nature, 282, 587

Melatos, A. 1997, MNRAS, 288, 1049 .1999, ApJ, 519, L77

Mereghetti, S., Israel, G. L., \& Stella, L. 1998, MNRAS, 296, 689

Michel, F. C., \& Goldwire, H. C. 1970, ApJ, 5, L21

Miller, M. C. 1995, ApJ, 448, L29

Murakami, T., Kubo, S., Shibazaki, N., Takeshima, T., Yoshida, A., \& Kawai, N. 1999, ApJ, 510, L119

Murakami, T., Tanaka, Y. Kulkarni, S. R., Ogasaka, Y. Sonobe, T., Ogawara, Y., Aoki, T., \& Yoshida, A. 1994, Nature, 368, 127

Neuhauser, D., Koonin, S. E., \& Langanke, K. 1987, Phys. Rev. A, 36, 4163

Novikov, I. D., \& Thorne, K. S. 1973, in Black Holes, ed. C. DeWitt \& B. DeWitt (New York: Gordon \& Breach), 343

Paczyński, B. 1992, Acta Astron., 42, 145

Reisenegger, A., \& Goldreich, P. 1992, ApJ, 395, 240

Rho, J., \& Petre, R. 1997, ApJ, 484, 828

Ruderman, M. A. 1991, ApJ, 382, 576

Ruderman, M. A., Zhu, T., \& Chen, K. 1998, ApJ, 492, 267

Schaeffer, R., Reeves, H., \& Orland, H. 1982, ApJ, 254, 688

Shaham, J. 1977, ApJ, 214, 251

Shakura, N. I., \& Sunyaev, R. A. 1973, A\&A, 24, 337

Smith, D. A., Bradt, H. V., \& Levine, A. M. 1999, ApJ, 519, L147

Thompson, C. 2000, in Highly Energetic Physical Processes and Mechanisms for Emission from Astrophysical Plasmas, ed. P. C. H. Martens \& S. Tsuruta (San Francisco: AIP), 245

Thompson, C., \& Blaes, O. 1998, Phys. Rev. D, 57, 3219 (TB98)

Thompson, C., \& Duncan, R. 1993, ApJ, 408, 194 (TD93)

1995, MNRAS, 275, 255 (TD95) 1996, ApJ, 473, 322 (TD96)

van Kerkwijk, M. H., Kulkarni, S. R., Matthews, K., \& Neugebauer, G. 1995, ApJ, 444, L33

Van Riper, K. A., Epstein, R. I., \& Miller, G. S. 1991, ApJ, 381, L47

Vasisht, G., \& Gotthelf, E. V. 1997, ApJ, 486, L129

Vasisht, G., Kulkarni, S. R., Frail, D. A., \& Greiner, J. 1994, ApJ, 431, L35

Wang, Z., Qu, Q., Luo, D., McCray, R., \& MacLow, M. 1992, ApJ, 388, 127

Woods, P. M., et al. 1999a, ApJ, 519, L139 1999 b, ApJ, 524, L55 (Paper I)

Woods, P. M., Kouveliotou, C., van Paradijs, J., Finger, M. H., \& Thompson, C. 1999c, ApJ, 518, L103 